\title{
ON THE INTERPRETATION OF THE AGE DISTRIBUTION OF STAR CLUSTERS IN THE SMALL MAGELLANIC CLOUD
}

\author{
MARK Gieles \\ Astronomical Institute, Utrecht University, 3584 CC Utrecht, Netherlands; and European Southern Observatory, \\ Santiago 19, Chile; mgieles@eso.org \\ Henny J. G. L. M. LAmers \\ Astronomical Institute, Utrecht University, 3584 CC Utrecht, Netherlands; lamers@astro.uu.nl \\ AND \\ Simon F. Portegies Zwart \\ Astronomical Institute “Anton Pannekoek," University of Amsterdam, 1098 SJ Amsterdam, Netherlands; \\ and Section Computational Science, University of Amsterdam, 1098 SJ Amsterdam, \\ Netherlands spz@science.uva.nl \\ Received 2007 January 15; accepted 2007 June 8
}

\begin{abstract}
We reanalyze the age distribution $(d N / d t)$ of star clusters in the Small Magellanic Cloud (SMC) using age determinations based on the Magellanic Cloud Photometric Survey. For ages younger than $3 \times 10^{9}$ yr the $d N / d t$ distribution can be approximated by a power-law distribution, $d N / d t \propto t^{-\beta}$, with $-\beta=-0.70 \pm 0.05$ or $-\beta=-0.84 \pm 0.04$, depending on the model used to derive the ages. Predictions for a cluster population without dissolution limited by a $V$-band detection result in a power-law $d N / d t$ distribution with an index of $\sim-0.7$. This is because the limiting cluster mass increases with age, due to evolutionary fading of clusters, reducing the number of observed clusters at old ages. When a mass cut well above the limiting cluster mass is applied, the $d N / d t$ distribution is flat up to $1 \mathrm{Gyr}$. We conclude that cluster dissolution is of small importance in shaping the $d N / d t$ distribution, and incompleteness causes $d N / d t$ to decline. The reason that no (mass independent) infant mortality of star clusters around $\sim 10-20 \mathrm{Myr}$ is found is explained by a detection bias toward clusters without nebular emission, i.e., clusters that have survived the infant mortality phase. The reason we find no evidence for tidal (mass dependent) cluster dissolution in the first gigayear is explained by the weak tidal field of the SMC. Our results are in sharp contrast to the interpretation of Chandar et al., who interpret the declining $d N / d t$ distribution as rapid cluster dissolution. This is due to their erroneous assumption that the sample is limited by cluster mass, rather than luminosity.
\end{abstract}

Subject headings: galaxies: individual (Small Magellanic Cloud) — galaxies: star clusters — stars: formation

Online material: color figures

\section{INTRODUCTION}

Star clusters are often assumed to be tracers of the star formation history of their host galaxy. Recent studies on the star formation rate in the solar neighborhood have revealed that the majority of stars form in a clustered environment (Clarke et al. 2000; Lada \& Lada 2003), while only a few percent of stars in the solar neighborhood are in clusters. Understanding the process of cluster dissolution is, therefore, of key importance if one wants to make a meaningful translation from the observed age distribution of star clusters to a star formation history of their host galaxy.

Recent theoretical work suggest that a large fraction $(50 \%-$ $90 \%$ ) of the star clusters disperse a few megayears after formation due to the expulsion of residual gas by the stellar winds and supernovae of massive stars (e.g., Goodwin 1997; Boily \& Kroupa 2003). If the star formation efficiency is independent of cluster mass $\left(M_{\mathrm{cl}}\right)$, then this "infant mortality" is also independent of $M_{\mathrm{cl}}$ (Goodwin \& Bastian 2006).

The observed age distribution of star clusters in M51 (Bastian et al. 2005) indicates that $~ 70 \%$ of the clusters disperse on a timescale of $\sim 10 \mathrm{Myr}$ after formation, roughly independent of $M_{\mathrm{cl}}$. Fall et al. (2005) claim that in the "Antennae" galaxies this process removes roughly $90 \%$ of the clusters each age dex during the first gigayear. This timescale is far too long to be explained by the gas expulsion scenario.
The $M_{\mathrm{cl}}$ independent dissolution due to gas expulsion is in sharp contrast to dissolution due to two-body relaxation in a tidal field (e.g., Chernoff \& Weinberg 1990; Vesperini \& Heggie 1997; Takahashi \& Portegies Zwart 2000; Baumgardt \& Makino 2003), or external perturbations by the disk (Ostriker et al. 1972; Gnedin \& Ostriker 1997) or giant molecular clouds (Spitzer 1958; Gieles et al. 2006b), which all have a more destructive effect on low-mass clusters than on high-mass clusters.

The observed age distributions of star clusters can be used to disentangle the two aforementioned disruption processes: massindependent or mass-dependent cluster dissolution. If the disruption time $\left(t_{\mathrm{dis}}\right)$ has a power-law dependence on the cluster mass, then the power-law index can be derived from the slope of the age distribution. This holds for mass-limited and magnitudelimited cluster samples, since at old ages disruption will usually dominate over evolutionary fading (Boutloukos \& Lamers 2003, hereafter BL03). On the other hand, in the case of a $M_{\mathrm{cl}}$ independent infant mortality process the age distribution of a masslimited cluster sample results in an age distribution of the form $d N / d t \propto t^{-1}$, where the index of -1 applies to the case in which $90 \%$ of the clusters dissolve each age dex (Fall et al. 2005; Whitmore et al. 2007). If the sample is magnitude limited, then the age distribution will be steeper. This is because fading and disruption both remove a certain fraction from the $d N / d t$ distribution. 
Recently, Chandar et al. (2006, hereafter CFW06) studied the age distribution of clusters in the Small Magellanic Cloud (SMC) based on cluster ages derived by Rafelski \& Zaritsky (2005, hereafter RZ05). They claim that the age distribution is consistent with a power law with index -1 for $t<3 \mathrm{Gyr}$ and that the sample is "reasonably complete down to $10^{3} M_{\odot}$ over this age range." They conclude that their observations are consistent with infant mortality being at work for $\sim 1 \mathrm{Gyr}$ and independent of $M_{\mathrm{cl}}$.

In this paper we address the interpretation of the $d N / d t$ distribution of star clusters in the SMC and the pitfall caused by detection incompleteness that can lead to the fallacious conclusion that mass-independent cluster disruption shapes the $d N / d t$ distribution.

The structure of the paper is as follows. In $\S 2$ we discuss the observations from which the SMC cluster sample was derived and the mass and age determinations of the clusters. Because the interpretation of the age distribution of the cluster sample depends heavily on the (in)completeness of the sample, we investigate different incompleteness scenarios in $\S 3$. In $\S 4$ we compare the resulting age distribution to predictions and argue that the shape of the age distribution can be explained by evolutionary fading, without involving mass-independent dissolution. In $\S 5$ we compare our results to other studies, and our conclusions are outlined in $\S 6$.

\section{OBSERVATIONS}

The clusters analyzed by RZ05 were identified by Hill \& Zaritsky (2006, hereafter HZ06). Photometric data in the UBVI bands from the Magellanic Clouds Photometric Survey (MCPS; Zaritsky et al. 1997) were used to find clusters. Stellar density images based on the photometric catalog of SMC stars were constructed by counting the number of stars with $V<20.5$ in squares of $10^{\prime \prime}$. We note that for the photometry all stars in the catalog were used, but only for clusters that were identified in the sample limited to stars with $V<20.5$.

RZ05 derived ages by comparing the $U-B, B-V$, and $V-I$ colors from clusters using Starburst99 (hereafter SB99; Leitherer et al. 1999) and Göttingen Galaxy Evolution Group (GALEV; Schulz et al. 2002; Anders \& Fritze-v. Alvensleben 2003) models. We adopt their ages, which have been derived from the photometry with the mean foreground extinction correction for the SMC of $E(B-V)=0.09$, but without a correction for local extinction. RZ05 try to include extinction as a free parameter in the age fitting method, but conclude that the scatter in the photometry is too large to improve the age estimates by including extinction as an additional parameter. They take extinction into account in an analytical way, ${ }^{1}$ but again conclude that the spread of observed clusters colors around the models does not reduce. Since this artificially imposed extinction as a function of age could introduce systematic trends in the $d N / d t$ distribution, combined with the conclusion of RZ05 that this method did not reduce the scatter of the cluster colors, we prefer the first-order age estimates of RZ05.

Adopting the ages of the clusters based on their photometry, we derived initial masses of the clusters, i.e., corrected for mass loss due to stellar evolution, independently using SB99 and GALEV models with $Z=0.004$. For the SB99 models we adopt identical settings as RZ05, i.e., standard mass loss, the full isochrone mass

\footnotetext{
1 RZ05 adopted the mean extinction measured by Harris \& Zaritsky (2004) for stars younger than $10 \mathrm{Myr}$ and older than 1 Gyr. For intermediate ages they interpolated the extinction value. RZ05 then corrected the colors for the clusters for which an age estimate is already available, and recalculated the best fit with the predicted cluster models.
}

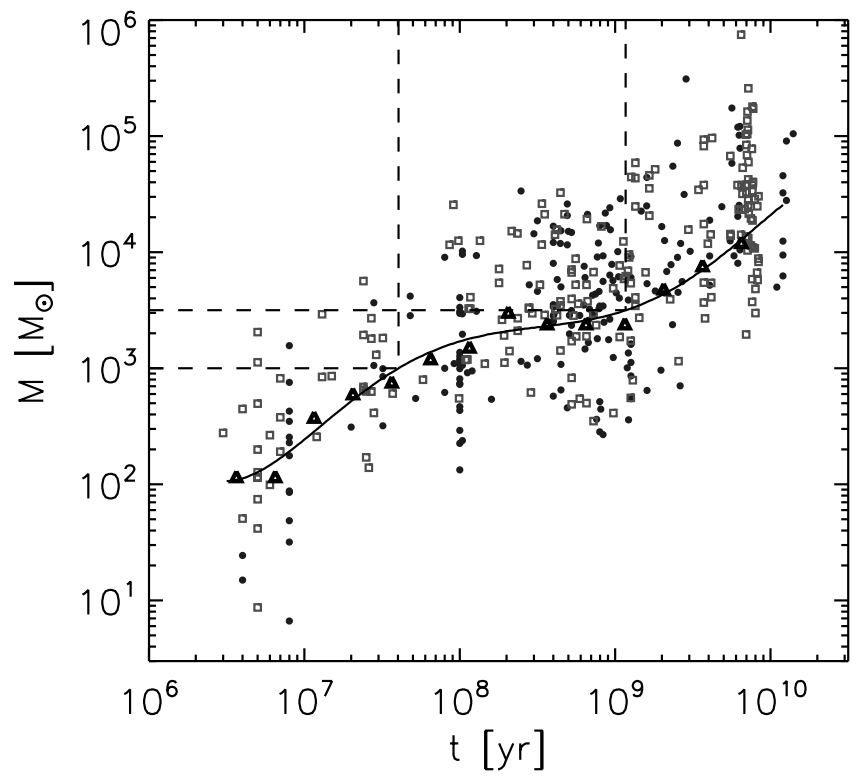

FIG. 1.- Age-mass diagram of 195 clusters in the SMC based on ages derived by RZ05 with the GALEV models (circles) and the SB99 models (squares). The $80 \%$ limit as a function of age $\left[M_{\mathrm{cl}}^{80 \%}(t)\right]$ is derived from the data (see text for details) and shown as triangles for SB99. A polynomial fit to $M_{\mathrm{cl}}^{80 \%}(t)$ is shown as a solid line. The vertical dashed lines indicate the ages where the $M_{\mathrm{cl}}^{80 \%}(t)$ curve intersects with $\log M_{\mathrm{cl}}=3$ and 3.5. [See the electronic edition of the Journal for a color version of this figure.]

interpolation, and a Salpeter initial mass function from 0.1 to $100 M_{\odot}$.

In Figure 1 we show the resulting age-mass diagram for the 195 clusters for which an age estimate is available. For both samples an increase in the upper and lower mass boundaries with age can be seen. The increasing upper mass boundary is due to a size of sample effect, since the $x$-axis is the logarithm of age. For a constant cluster formation rate, more clusters are formed in each dex for increasing $\log t$. For a power-law cluster initial mass function (CIMF) with index -2 the upper boundary is expected to increase as a straight line with slope +1 (Hunter et al. 2003; Gieles et al. 2006a), in reasonable agreement with Figure 1.

The sloping lower mass boundary is due to incompleteness, most likely because of evolutionary fading of clusters with age. Figure 1 clearly shows that the limiting cluster mass in the sample of RZ05 is not constant, contrary to the assumption made by CFW06 in their analysis of the age distribution. Note that CFW06 do not present an age-mass diagram in their work to support their assumption, but rely on the assumption that the sample "is likely to be approximately surface brightness limited, rather than luminosity limited." In $\S 3$ we investigate the possible causes for incompleteness and the effect on the $d N / d t$ distribution in more detail.

Because of the method used by HZ06 to define the cluster sample, the sample of RZ05 is not expected to be magnitude limited only (see $\S 3$ ). In an attempt to quantify the increasing lower $M_{\mathrm{cl}}$ as a function of age, we empirically derive the mass limit above which $80 \%$ of the clusters are more massive $\left[M_{\mathrm{cl}}^{80 \%}(t)\right]$. The limit is chosen at $80 \%$, since we find this to be the point where the mass function (at different ages) turns over. This suggests the sample is relatively complete above this limit and highly incomplete below it.

At intervals of 0.25 dex in $\log t$ we count the number of clusters in a bin with a width of 0.5 dex. The masses in the bin are sorted, and we count from the highest mass until we have reached $80 \%$ of the total number in the bin. Note that this $M_{\mathrm{cl}}^{80 \%}(t)$ is not 
the same as an $80 \%$ completeness limit, or the detection limit, as usually derived from artificial cluster experiments, since we here have no information about clusters that did not make it into the sample. However, if we assume that the shape of the completeness curve (i.e., the function that describes the fraction of clusters that is retrieved as a function of mass) is not dependent on age, we can safely assume that the evolution of $M_{\mathrm{cl}}^{80 \%}$ with age is the same shape as that for the completeness limit.

The result of $M_{\mathrm{cl}}^{80 \%}(t)$ is shown as triangles in Figure 1. A polynomial fit is shown as a solid line. Note that the curve is located relatively high above the lower limit. If the sample had a "hard" detection limit and the cluster IMF had an index of -2 and the cluster dissolution would be mass independent, we would expect that at each age the $M_{\mathrm{cl}}^{80 \%}(t)$ line is only a factor of 1.25 higher in mass than the lower limit. Figure 1 shows that the $80 \%$ limit is at about an order of magnitude higher mass than the absolute lower limit. This implies the data are highly incomplete for low-mass clusters. In $\S 3$ we compare the $M_{\mathrm{cl}}^{80 \%}(t)$ curve to different predictions.

\section{INCOMPLETENESS: LIMITED BY MASS, BY LUMINOSITY, OR A COMBINATION OF BOTH?}

The interpretation of the empirical age distribution of the SMC clusters depends strongly on how incompleteness affects the sample. Therefore, we compare the empirically derived $M_{\mathrm{cl}}^{80 \%}(t)$ curve to what is expected from the selection procedure of HZ06 and RZ05.

\subsection{A Sample Limited by the Number of Stars in a Cluster}

From the stellar catalog HZ06 selected stars with $V<20.5$ mag. They constructed number density images from which overdensities were detected. This implies that only clusters with enough stars with $V<20.5$ could end up in the catalog.

We first assume that the number of stars with $V<20.5[N(V<$ $20.5)]$ determines the limiting cluster mass. We use the evolutionary isochrones of the Padova models for $Z=0.004$ (Bertelli et al. 1994; Girardi et al. 1996, 2000), which were converted to the UBVRIJHK photometric system by Girardi et al. (2002). We assume a Salpeter stellar initial mass function (IMF) with lower mass of $0.5 M_{\odot}$. Stars below this mass do not reach $V<20.5$ within the Hubble time. The number of stars with $V<20.5$ is counted for cluster models of different ages and masses, and we select only clusters with $N(V<20.5)$ greater than some fixed number. We adopted $N(V<20.5)=25$ because the resulting mass-age relation agrees more or less with the location of the $M_{\mathrm{cl}}^{80 \%}(t)$ line in Figure 2.

In Figure 2 we show the result as a dotted line. The curve is slowly increasing for $\log t \lesssim 9$ and then rises. This transition occurs at the age where the main-sequence turnoff drops below $V=20.5$. Below that age a cluster of a given mass has nearly constant $N(V<20.5)$, since the brightness of stars on the main sequence is not varying much and the fraction of stars in $N(V<$ $20.5)$ that is on the main sequence is high. When the turnoff drops below $V=20.5, N(V<20.5)$ consists of red giant branch and asymptotic giant branch (RGB and AGB) stars mainly. Because the fraction of RGB and AGB stars in a cluster is small, but is responsible for the majority of the cluster luminosity, the minimum mass of a cluster with $N(V<20.5)=25$ steeply increases with age.

\subsection{A Sample Limited by the Total Cluster Luminosity}

After HZ06 selected their clusters from the stellar density image, as described in $\S 2$, a visual inspection on the original im-

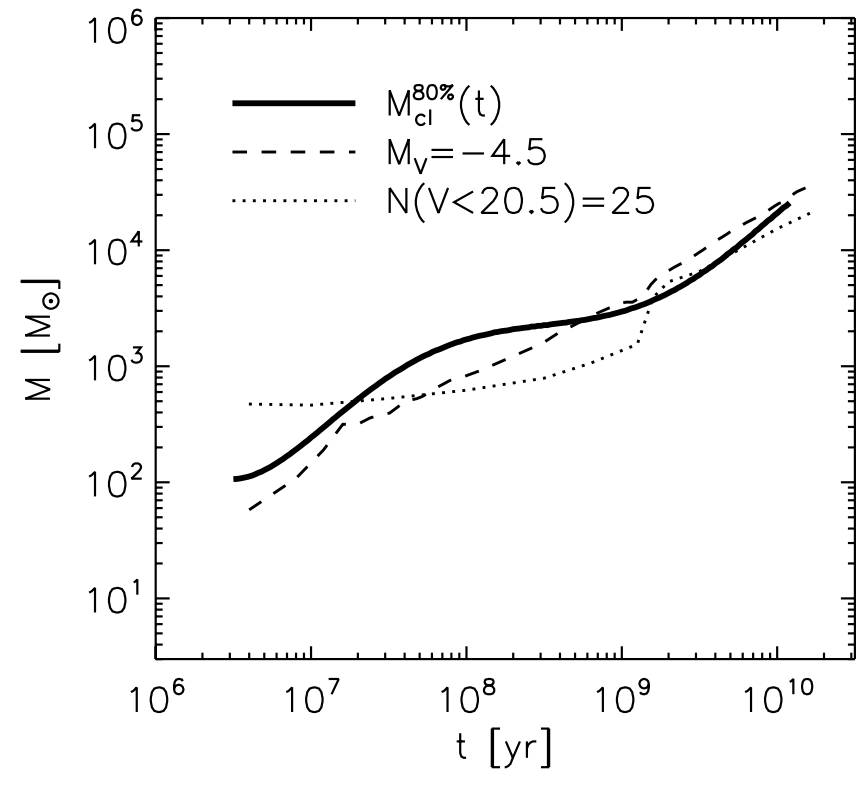

FIG. 2.-Empirically derived $M_{\mathrm{cl}}^{80 \%}(t)$ curve above which $80 \%$ of the clusters are located as derived in $\S 2$ and Fig. 1 (solid line). Also shown are the two predicted lines for the cluster models with a limiting mass evolution based on the assumption of a constant number of stars brighter than $V<20.5$ (dotted line) and on the assumption of a limiting cluster magnitude at $M_{V}<-4.5$ (dashed line).

ages was done. Only the unambiguous clusters were kept. After this, they fitted surface brightness profiles to the remaining sources. Only when a reliable surface brightness fit and photometry could be extracted, the source was kept in their sample and called a "star cluster." The total number of sources found from the stellar density images was a factor of 4 higher than the final number of star clusters in the catalog (D. Zaritsky 2006, private communication). This indicates that accurate photometry was a strongly limiting factor in the selection of the clusters. Since this last selection step relies on the total luminosity of a cluster, we may assume that the sample is mainly limited by the magnitude of the clusters. To test this assumption, we predict what is expected for the limiting mass as a function of age for a constant magnitude limit.

Star clusters are formed on a timescale short enough that their photometric evolution can be well described by simple stellar population (SSP) models. From these models it follows that the flux of a star cluster scales approximately as a power law with age $\left(F_{\lambda} \propto t^{-\zeta}\right)$, with $\zeta$ positive. The limiting cluster mass as a function of age $\left[M_{\mathrm{cl}}^{\lim }(t)\right]$ of a sample that is limited by an absolute magnitude at a certain wavelength $\left(M_{\lambda}^{\lim }\right)$ can be expressed in the magnitude evolution from an SSP model $\left[M_{\lambda}^{\mathrm{SSP}}(t)\right]$ :

$$
\log M_{\mathrm{cl}}^{\lim }(t)=\log M_{\mathrm{cl}, i}^{\mathrm{SSP}}-0.4\left[M_{\lambda}^{\lim }-M_{\lambda}^{\mathrm{SSP}}(t)\right],
$$

where $M_{\mathrm{cl}, i}^{\mathrm{SSP}}$ is the initial mass of the cluster that is described by the SSP model. For the GALEV and SB99 version that we use, $M_{\mathrm{cl}, i}^{\mathrm{SSP}}=10^{6} M_{\odot}$. When taking $M_{\mathrm{cl}, i}^{\mathrm{SSP}}$ as a constant $M_{\mathrm{cl}}^{\lim }(t)$ represents an initial mass, i.e., before mass loss due to stellar evolution.

Since $\log M_{\mathrm{cl}, i}^{\mathrm{SSP}}(t)$ and $M_{\lambda}^{\lim }$ from equation (1) are constant and $0.4 M_{\lambda}^{\mathrm{SSP}} \propto-\log F_{\lambda}=\zeta \log t$, we can rewrite equation (1) as

$$
\log M_{\mathrm{cl}}^{\lim }(t)=\zeta \log t+C,
$$

with $C \equiv \log M_{\mathrm{cl}, i}^{\mathrm{SSP}}-0.4 M_{\lambda}^{\lim }$ (BL03).

In Figure 2 we show the result of equation (1) as a dashed line for the SB99 models with an adopted limiting magnitude of 

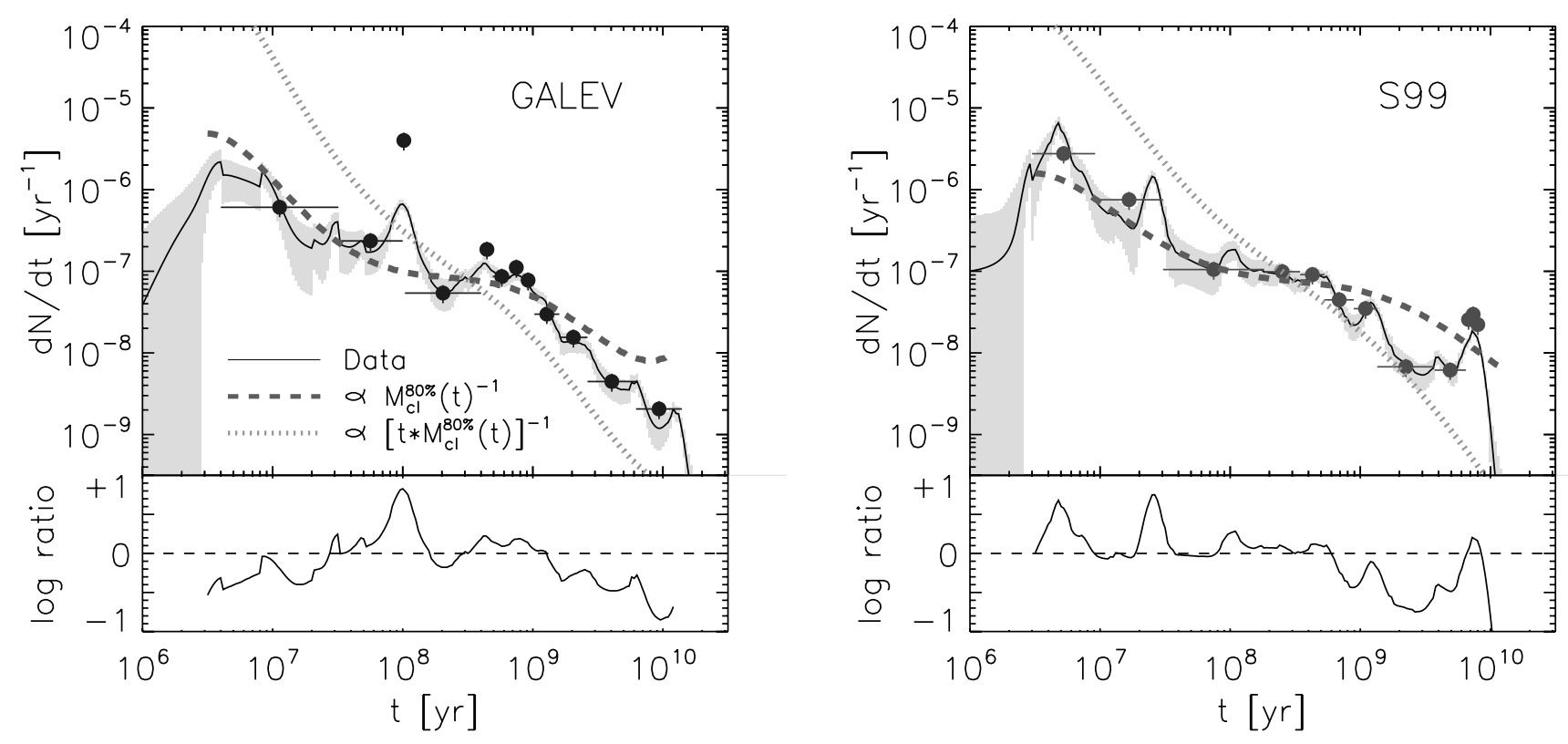

FIG. 3.-Top panels: Age or $d N / d t$ distribution from GALEV (left) and SB99 (right) results. Solid line: Each cluster was represented by a Gaussian profile with $\sigma_{ \pm}$corresponding to the minimum and maximum uncertainty in the age determination. The gray area corresponds to the $1 \sigma$ uncertainty. Circles with error bars: Age distribution shown as a histogram where the horizontal lines indicate the variable width of the age bins, where each bin contains 17 clusters (see text for details). Thick dashed line: Predicted $d N / d t$ distributions for a sample limited by $M_{\mathrm{cl}}^{80 \%}(t)$ of Fig. 1. The thick dotted line represents a cluster sample limited by $M_{\mathrm{cl}}^{80 \%}(t)$ combined with the mass-independent disruption model, which was suggested by CFW06. This distribution declines much more rapidly than the data. Bottom panels: Ratio between the observed $d N / d t$ distribution and the predicted one for the sample limited by $M_{\mathrm{cl}}^{80 \%}(t)$. [See the electronic edition of the Journal for a color version of this figure.]

$M_{V}^{\lim }=-4.5$. This value was chosen because the resulting limiting age-mass relation agrees more or less with the location of the empirically derived $M_{\mathrm{cl}}^{80 \%}(t)$ limit of Figures 1 and 2 . Note that this prediction matches the empirically derived $M_{\mathrm{cl}}^{\lim }(t)$ quite well. We especially note that this predicted relation fits the empirical one much better than the $N(V<20.5)$ curve.

We conclude that the cluster sample of RZ05, used to derive the age distribution of SMC clusters, is mainly magnitude limited.

\section{THE AGE DISTRIBUTION OF SMC CLUSTERS}

\subsection{The Observed Age Distribution}

The uncertainties in the age determinations of RZ05 are quite large, mainly due to the photometric uncertainty of faint clusters. We take these uncertainties into account when constructing the $d N / d t$ distribution.

We represent the contribution by each cluster to the age distribution as an asymmetric Gaussian, with the lower width $\left(\sigma_{-}\right)$ and the upper width $\left(\sigma_{+}\right)$corresponding to the minimum and maximum age $\left(t_{-}\right.$and $t_{+}$) derived by RZ05. RZ05 based these latter values on the $90 \%$ confidence range from their $\chi^{2}$ results of the photometric age determination. This corresponds to $1.6 \sigma$. We assume a Gaussian spread in $\log t$, since the errors in the age determination are roughly constant in $\log t$ (mean $\Delta \log t \simeq 0.25$ ). The definition of $\sigma_{ \pm}$is then $\sigma_{ \pm} \equiv\left|\log t_{ \pm}-\log t\right| / 1$.6. This results in an asymmetric Gaussian profile for each cluster, where the left and right side both have a surface of 0.5 , such that the total contribution of each cluster to the age distribution is 1 . Some ages derived with SB99 have $\sigma_{-}=0$ or $\sigma_{+}=0$. In that case we adopted a minimum uncertainty of $\sigma_{ \pm}=0.05$. When all clusters are added in a large $\log t$ array of equal intervals in $\log t$, each value of $d N$ (i.e., the number of clusters in that age interval) is divided by the width of the bin in linear age, to construct the $d N / d t$ distribution.

In the left and right panels of Figure 3 we present the smoothed $d N / d t$ result for the GALEV and SB99 modeling, respectively. The solid line represents the full data set. We estimated Poisson errors by counting the number of clusters in bins of width $0.25 \mathrm{dex}$, corresponding to the mean uncertainty in the log age values of RZ05. The $1 \sigma$ Poisson errors are shown as a gray shaded region. Note that we do not normalize the cluster age distribution to the stellar age distribution, as was done by RZ05. This is done to compare our results to the results of CFW06, who also construct the unnormalized cluster age distribution.

This representation of the $d N / d t$ distribution takes into account the increasing age interval for the increasing width of the age intervals. The number of clusters per logarithmic age bin actually slightly increases with $\log t$, which reflects the small increase in number density that can be seen in Figure 1. Note that $d N / d t \propto t^{-1}$ implies a constant number of clusters in constant $\log t$ bins.

In Figure 3 we also show a binned $d N / d t$ histogram as circles with small error bars. The sizes of the bins are chosen such that there are 17 clusters in each bin (following Maíz Apellániz \& Úbeda 2005). This results in a total of 10 bins over a range of $\sim 4.5$ age dex. The obtained average bin width is then 0.35 dex, which is larger than the mean error in the age determination making it suitable for a fit where we only take into account the uncertainties in the direction of the $y$-axis. The first bin starts at the age of the youngest cluster, and the last bin ends at the age of the oldest cluster. This way of binning the data results in a $d N / d t$ distribution very similar to the Gaussian smoothed version (Fig. 3).

We fit a straight line to $\log (d N / d t)$ versus $\log t$ for $t<3 \mathrm{Gyr}$ using a $\chi^{2}$-error statistics minimization with bin weights $\left(W_{i}\right)$ depending on the standard deviation of each bin $\left(\sigma_{i}\right)$ as $W_{i}=$ $1 / \sigma_{i}^{2}$, which for this representation is $W_{i}=\ln ^{2}(10) N_{i}$. We find a slope, i.e., the power-law index, of $-0.70 \pm 0.05$ if we use the GALEV results, and $-0.84 \pm 0.04$ if we use the SB99 result. The mean index is thus $0.77 \pm 0.07 .^{2}$

\footnotetext{
2 If we ignore the bin weights in the fit, we find uncertainties on the indices of 0.20 and 0.08 for the fits on GALEV and SB99, respectively. This could be the explanation for the large uncertainty of 0.15 found by CFW06 in their index of 0.85 .
} 
We conclude that the observed age distribution in the age range of $7 \lesssim \log t \lesssim 9.5$ can be approximated by a power law with index $-0.77 \pm 0.07$.

\subsection{The Predicted Age Distribution}

Assume that a cluster population formed at a constant rate and with a power-law CIMF with index $-\alpha$. Then, if there is no dissolution, the age distribution of all clusters can be acquired by integrating over all masses from $M_{\mathrm{cl}}^{\mathrm{lim}}$ (from eqs. [1] or [2]) to $M_{\mathrm{cl}}^{\max }(\mathrm{BL03})$ :

$$
\begin{aligned}
d N / d t & =\int_{M_{\mathrm{cl}}}^{M_{\mathrm{cl}}^{\max }} S M_{\mathrm{cl}}^{-\alpha} d M_{\mathrm{cl}} \\
& =\frac{S}{1-\alpha}\left[\left(M_{\mathrm{cl}}^{\max }\right)^{1-\alpha}-\left(M_{\mathrm{cl}}^{\lim }\right)^{1-\alpha}\right] \\
& \propto 1 / M_{\mathrm{cl}}^{\lim }
\end{aligned}
$$

where $S$ describes the cluster formation rate. In the last steps we have used $\alpha=2$ and $M_{\mathrm{cl}}^{\max } \gg M_{\mathrm{cl}}^{\lim }$. If the sample is magnitude limited, as we have shown in $\S 3$, with $M_{\mathrm{cl}}^{\lim } \propto t^{\zeta}$, then the age distribution scales with $t$ as $d N / d t \propto t^{-\zeta}$. So we expect a slope $-\zeta$ in the logarithmic representation of the $d N / d t$ distribution (Fig. 3). On the other hand, for a sample that is mass limited (corresponding to $M_{\mathrm{cl}}^{\text {lim }}$ being constant) without dissolution the age distribution would be flat.

The value of $\zeta$ can be determined by approximating $\log M_{\mathrm{cl}}^{\mathrm{SSP}}(t)$ versus $\log t$ following from SSP models by a straight line. For ages smaller than $3 \mathrm{Gyr}$, the value of $\zeta$ derived from the GALEV models are $[1.02,0.89,0.72,0.63$, and 0.58$]$ for $U, B, V, R$, and $I$, respectively. For the SB99 models we find [0.93, 0.78, 0.66, 0.60 , and 0.54$]$ for the same filters, respectively. So, for a cluster sample formed with constant formation rate, not affected by disruption and limited by a detection in the $V$ band, the predicted age distribution is a power law with index $\sim-0.7$, in agreement with the observations $(\S 4.1)$.

Using the empirically derived $M_{\mathrm{cl}}^{\lim }(t)$ from Figures 1 and 2, we predict the $d N / d t$ distribution for a cluster sample that is not affected by any disruption and thus declines as $\left[M_{\mathrm{cl}}^{80 \%}(t)\right]^{-1}$ (thick dashed lines in Fig. 3). The prediction and the observed data are normalized at $\log t=8.5$. The prediction describes the overall decline and the details in the shape of the observed $d N / d t$ distribution very well. This suggests that there are no signs for cluster disruption present in this data set, and the declining $\mathrm{dN} / \mathrm{dt}$ distribution can be explained by incompleteness only.

To illustrate the combined effect of disruption and incompleteness, we also show the $d N / d t$ distribution for a sample where we apply the $90 \%$ reduction of clusters each age dex, as suggested by CFW06 (thick dotted line), also normalized to the data at $\log t=8.5$. We stress that the mass-independent disruption model of CFW06 works in addition to incompleteness, such that both make the $d N / d t$ distribution steeper. Therefore, a fallacious assumption that a cluster sample is mass limited, while it is in reality luminosity limited, will lead to confusion between disruption and incompleteness.

The nice agreement between the observed $d N / d t$ distribution and the $\left[M_{\mathrm{cl}}^{80 \%}(t)\right]^{-1}$ curve implies that the observed age distribution of the SMC clusters with ages $\log t / \mathrm{yr} \lesssim 9.5$ can be explained by evolutionary fading only, without the need for mass-independent dissolution. The fact that there is no evidence for infant mortality at young ages $(\$ 30 \mathrm{Myr})$ is most likely due to a bias of clusters without nebular emission (HZ06), which typically have such young ages. We expect that the effect of gas removal must de- stroy young (few megayears) clusters in the SMC, but we conclude that the sample under discussion here is not suitable to investigate this phenomenon in detail. In a future study we will investigate the infant mortality rate of young star clusters in the SMC using different techniques (M. Gieles et al. 2008, in preparation).

Also, tidal evaporation does not affect the $d N / d t$ distribution for SMC clusters with ages $\lesssim 3$ Gyr. This agrees with the long survival times ( 26 Gyr for masses $\geq 10^{4} M_{\odot}$ ) predicted by $N$-body simulations of clusters dissolving in weak tidal fields (e.g., Baumgardt \& Makino 2003).

\subsection{The Age Distribution of a Mass-Limited Subsample}

The results of CFW06 are based on the assumption that the RZ05 sample of clusters is mass limited. To show the effect of a mass limit on the age distribution we compare the cumulative age distribution of the clusters with and without mass limit to the predictions. Such a cumulative distribution is preferred over constructing a histogram when only a small number of clusters is available, as is the case when applying a (relatively high) mass cut (see Fig. 1).

Figure 4 shows the cumulative age distributions divided by the age range from the minimum age in the sample $\left(t_{\min }\right): N\left(t^{\prime}<\right.$ $t) /\left(t-t_{\min }\right)$. This representation has the advantage that it resembles a $d N / d t$ distribution. The full SMC cluster sample is shown as thick solid lines, and the subsample of clusters with masses above $3 \times 10^{3} M_{\odot}$ is shown as thick dashed lines, using the masses and ages derived by using the GALEV models (left) and the SB99 models (right). The gray areas of each distribution indicate the $1 \sigma$ uncertainty due to Poisson statistics. Note that the subsample with the mass cut is flat up to $\sim 1 \mathrm{Gyr}$, while the full sample is declining. Signs of flattening are also present in the $d N / d t$ histogram of CFW06 (their Fig. 1) for a subsample with $M>$ $10^{3} M_{\odot}$. The authors also note this flattening and argue that "the mass-limited sample is somewhat shallower than the one constructed from the entire sample, since there are relatively few clusters more massive than $10^{3} M_{\odot}$ at very young ages." While this is indeed the correct explanation for why the $d N / d t$ distribution of a mass-limited subsample is flatter, it should not be the case in their suggested scenario. In their scenario, the number of clusters in $\log t$ bins of equal width should be constant. Using the GALEV results, we find 36 clusters with masses above $3 \times 10^{3} M_{\odot}$, and ages between $10^{8.75}$ and $10^{9.25} \mathrm{yr}$, while there are zero clusters above this mass limit with ages between $10^{6.75}$ and $10^{7.25} \mathrm{yr}$. According to the model of CFW06 these numbers should be the same. In addition, we show in Figure 1 that a mass cut at $10^{3} M_{\odot}$ is not high enough to be safely above the increasing limiting mass up to 1 Gyr. A mass cut at $3 \times 10^{3} M_{\odot}$ would be a safer choice. Therefore, we will consider this limit.

We compare these cumulative distributions with those predicted for the power-law fits to $d N / d t$. If $d N / d t=C t^{-\eta}$, then the $N\left(t^{\prime}<t\right)$ depends on $t$ as

$$
N\left(t^{\prime}<t\right)=\frac{C}{1-\eta}\left(t^{1-\eta}-t_{\min }^{1-\eta}\right), \quad \text { if } \eta \neq 1 .
$$

We adopt $t_{\min }=3 \mathrm{Myr}$, since this is the youngest age present in the SSP models.

In $\S 4.1$ we have shown that for the full sample $\eta=0.70$ and 0.84 for GALEV and SB99, respectively. The resulting predicted cumulative distribution (eq. [4]), again normalized to $\left(t-t_{\min }\right)$, is shown by the thin solid line. Note that it agrees with the empirical distribution within about $2 \sigma$. For a sample that is mass limited above the detection limit and does not lose clusters by 

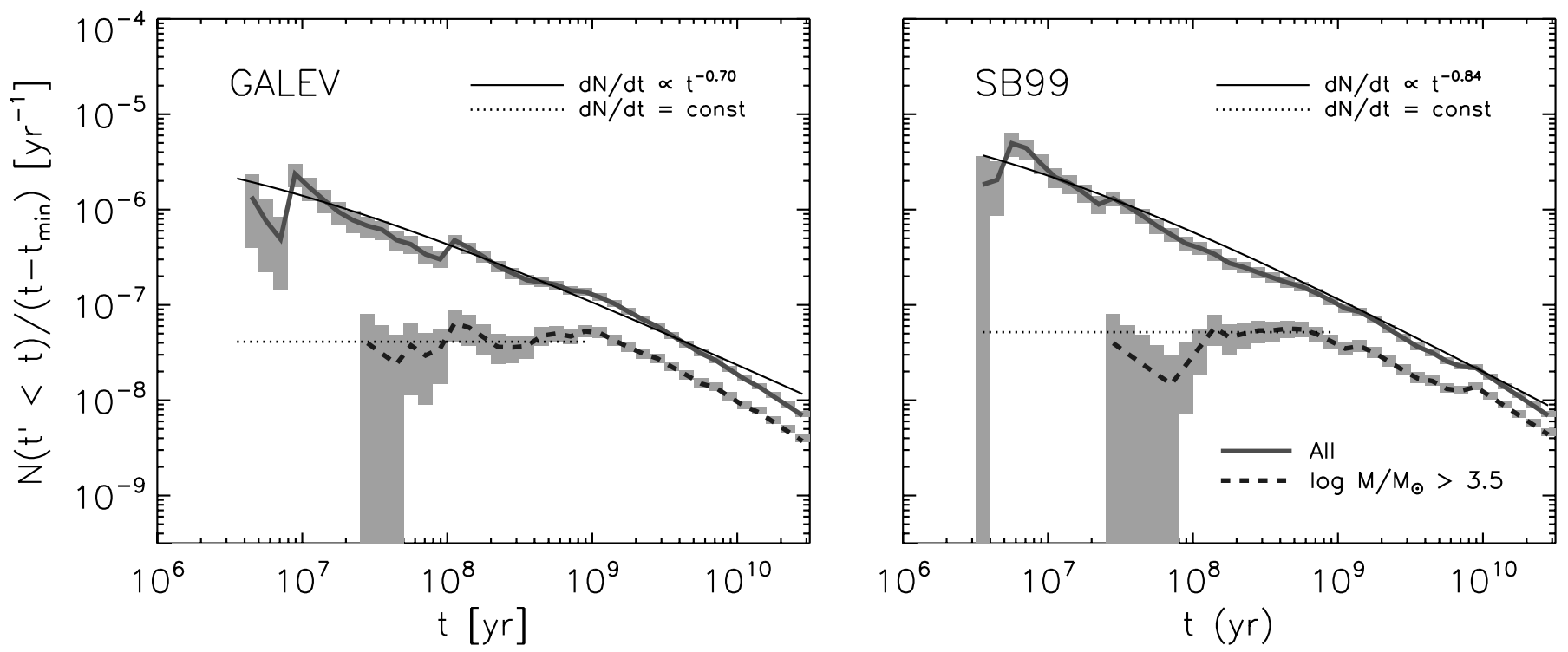

FIG. 4.-Cumulative age distribution $\left[N\left(t^{\prime}<t\right)\right]$ normalized to $\left(t-t_{\min }\right)$ for the GALEV $(l e f t)$ and SB99 $(r i g h t)$ results. The total sample is shown with thick solid lines, with the results of the power-law fits overplotted as thin lines. Eq. 4 was used to relate the power-law function to $N\left(t^{\prime}<t\right)$. The resulting distributions after a mass cut at $\log M=3.5$ is applied are shown as thick dashed lines. The predictions for a flat $d N / d t$ distribution, up to the age for which the sample is expected not to be affected by incompleteness ( $\sim$ Gyr; see Fig. 1), are also flat in this representation (see text and eq. 4 for details) and are shown as dotted lines. [See the electronic edition of the Journal for a color version of this figure.]

dissolution, i.e., $\eta=0$, the expected distribution will be flat in the representation of Figure 4. The thin dotted lines in Figure 4 show the predicted distributions. Again, these agree with the observed cumulative distributions up to an age of $\sim 1$ Gyr, corresponding to the maximum age for which the sample of clusters with $\log M=3.5$ is not affected by the $M_{\mathrm{cl}}^{80 \%}(t)$ line (Fig. 1). Beyond this age the distribution declines in a similar way as the full distribution, which is because even for the relatively high mass cut, the sample is affected by incompleteness in the same way as the full sample.

\section{COMPARISON TO OTHER STUDIES}

We first compare our results to other studies on the SMC star clusters. This is particularly interesting, since there appears to be a large variation between the different data sets and their interpretations.

One of the first detailed analyses of the age distribution was done by Hodge (1987). The age distribution presented in that work looks quite different from the one shown here and in RZ05 and CFW06. It is flat up to almost $1 \mathrm{Gyr}$ and then has a steep drop. This is quite similar to what is found in a recent study by Chiosi et al. (2006; their Fig. 10), who also present an age distribution for SMC clusters that is nearly flat the first $\sim 10^{8} \mathrm{yr}$. Hodge (1987) detects stars down to $B \simeq 22-23$, and Chiosi et al. (2006) even down to $V \simeq 24$. Both studies derive their ages with isochrone fitting techniques. This makes it possible to determine the age even if only a few stars are available. Assuming that at young ages ( $\lesssim$ few $100 \mathrm{Myr}$ ) all clusters are detected, a flat distribution is expected until the age where the fading or disruption starts to remove clusters from the sample. Using the sample of Hodge (1987), BL03 argued that the dissolution time of clusters in the SMC is very long and of order $8 \mathrm{Gyr}$ for a cluster of $10^{4} M_{\odot}$. This agrees with the fact that we do not find evidence for dissolution up to ages of about $3 \mathrm{Gyr}$ from the RZ05 sample. We note that Chiosi et al. (2006) present two age distributions. The one we refer to here is for clusters that were classified C (=genuine star clusters) by Bica $\&$ Dutra (2000). In their Figure 7 Chiosi et al. (2006) also present an approximately flat age distribution, defined as $d N / d \log t$, which corresponds to $d N / d t \propto t^{-1}$. However, we note that this sample includes all large OB associations, with sizes much larger $(\gtrsim 20 \mathrm{pc})$ than typical star clusters $(\sim 5 \mathrm{pc})$.

HZ06 and RZ05 consider only stars with $V<20.5$ for the selection of their clusters. This cut in the stellar sample is $\sim 2-3 \mathrm{mag}$ brighter than the one on the stellar sample mentioned before. In addition, the broadband photometry of RZ05 was based on King (1962) and Elson et al. (1987) profile fits to the surface brightness profile of clusters. More stars are needed for a good profile fit to the surface brightness profile than for an isochrone fit, which results in less clusters in the sample of RZ05 than in the samples of Elson et al. (1987) and Chiosi et al. (2006). This idea is supported by the larger number of clusters found in the deeper studies of Hodge (1987) and Chiosi et al. (2006), viz., 327 and 311, respectively. This supports our conclusion that incompleteness effects are more important in the sample of RZ05.

Comparing our results to those of CFW06, based on the same cluster sample of RZ05, we note that our interpretation of the age distribution is drastically different. CFW06 assumed that the cluster sample of RZ05 is mass limited. They fit the age distribution of the clusters with a power law of index $-0.85 \pm 0.15$, and argue that this is consistent within $1 \sigma$ with mass-independent dissolution in which the number of clusters decreases by a factor of 10 age-dex $^{-1}$, up to an age of about 3 Gyr. We have shown that the index of the age distribution is $-0.77 \pm 0.07(\S 4.1)$, i.e., consistent with -1 only within $3.3 \sigma$. Moreover (and more importantly), we have shown in $\S 2$ that the cluster sample is not mass limited, as is already clear from the distribution of the clusters in the age-mass diagram of Figure 1. We have given arguments that the cluster sample is approximately magnitude limited. This follows both from the way in which RZ05 have defined their cluster sample and from the observed 80\% limit of the stars in Figure 1. This limit has a very similar slope as that expected for a sample that is limited by the visual magnitude (Fig. 2). The observed index of $-0.77 \pm 0.07$ is very similar to the one predicted for a sample that is magnitude limited in the $V$ band $(0.72$ for GALEV models and 0.66 for SB99 models), if the number of clusters decreases only by evolutionary fading below the magnitude limit. 
We thereby rule out the need for the infant mortality scenario that proceeds up to $3 \mathrm{Gyr}$, as interpreted by CFW06.

From theoretical arguments it is expected that infant mortality works on a much shorter timescale $(\sim 10-20 \mathrm{Myr})$, due to the removal of gas that has not been used to form stars (e.g., Goodwin 1997; Boily \& Kroupa 2003). The lack of a strong bump in the age distribution of the SMC clusters at young ages ( $\leqslant 30 \mathrm{Myr}$ ), as was found for the clusters in M51 (Bastian et al. 2005) and the Antennae galaxies (Fall et al. 2005), is probably because the SMC sample of HZ06 has a bias toward clusters without strong nebular emission.

\section{CONCLUSIONS}

We conclude that the observed age distribution of the RZ05 sample of the SMC clusters agrees with that predicted for a cluster sample that is magnitude limited and in which the age distribution in terms of $d N / d t$ is decreasing with age by evolutionary fading of the clusters. There is no need to invoke a mass-independent infant mortality that extends to 3 Gyr and destroys $90 \%$ of the clusters every age dex, as suggested by CFW06. In fact, for a magnitude-limited cluster sample with an extended independent mass infant mortality, the age distribution would decrease more steeply with age than observed.

Interestingly, our result also explains the nearly flat age distribution of the field stars by RZ05, because not many clusters are dissolved between $10^{7}$ and $10^{9} \mathrm{yr}$, i.e., after the infant mortality phase. This is in contrast to the interpretation by CFW06 of a very long mass-independent disruption phase, because that model predicts a rapid increase in the age distribution of the field stars due to the continuing contribution of stars from dissolving clusters to the field. Such an increasing age distribution of the field stars is not observed.

In this paper we demonstrate that the interpretation of the cluster age distribution is very sensitive to incompleteness effects as a function of cluster age and to how these are taken into account. For extragalactic (slightly resolved) star clusters various tests methods have been developed to quantify (in)completeness and its dependence on cluster luminosity and radius (see, e.g.,
Bastian et al. 2005; Mora et al. 2007). For nearby cluster samples such as that of the SMC discussed here, it should be possible to construct completeness curves as a function of structural parameters of the clusters as well. By simulating star clusters of different ages and masses, thereby taking into account the luminosity evolution of the individual stars, and adding them to the catalogs, one can retrieve the artificial clusters from the catalog using the exact same cluster selection procedure as used for the data. This procedure is beyond the scope of this paper, since we mainly want to demonstrate that there is a degeneracy between incompleteness effects and the mass-independent disruption model of CFW06.

An important consequence of our result is that it confirms that cluster lifetimes are strongly dependent on the environment in which they evolve, which was already suggested by Hodge (1987) based on the difference in the age distribution for clusters in the SMC and the solar neighborhood (see also Lamers et al. [2005] for a comparison of cluster lifetimes in four galaxies). In contrast to this, Whitmore et al. (2007) use the result of CFW06 to show that the cluster $d N / d t$ distributions in the Antennae galaxies and in the SMC are very similar. Although it would simplify things if all clusters evolve similarly the first gigayear, it is rather counterintuitive, since then the evolution would not depend at all on the strength of the tidal field and the number of giant molecular clouds. Our results support a scenario in which the lifetime of clusters that have survived the gas removal phase is determined mainly by environmental factors.

We thank Bruce Elmegreen, Nate Bastian, and Søren Larsen for discussions and comments that improved the paper. We thank the referee, Dennis Zaritsky, for discussion on the cluster selection procedure and completeness. We are grateful to Evghenii Gaburov for help and discussions on fitting. This research was supported in part by the Netherlands Organization for Scientific Research (NWO grant 643.200.503), the Netherlands Advanced School for Astronomy (NOVA), the Royal Netherlands Academy for Arts and Sciences (KNAW), and the Leids Kerkhoven-Bosscha fonds ( $\mathrm{LKBF})$.
Anders, P., \& Fritze-v. Alvensleben, U. 2003, A\&A, 401, 1063

Bastian, N., Gieles, M., Lamers, H. J. G. L. M., Scheepmaker, R. A., \& de Grijs, R. 2005, A\&A, 431, 905

Baumgardt, H., \& Makino, J. 2003, MNRAS, 340, 227

Bertelli, G., Bressan, A., Chiosi, C., Fagotto, F., \& Nasi, E. 1994, A\&AS, 106, 275

Bica, E., \& Dutra, C. M. 2000, AJ, 119, 1214

Boily, C. M., \& Kroupa, P. 2003, MNRAS, 338, 673

Boutloukos, S. G., \& Lamers, H. J. G. L. M. 2003, MNRAS, 338, 717 (BL03)

Chandar, R., Fall, S. M., \& Whitmore, B. C. 2006, ApJ, 650, L111 (CFW06)

Chernoff, D. F., \& Weinberg, M. D. 1990, ApJ, 351, 121

Chiosi, E., Vallenari, A., Held, E. V., Rizzi, L., \& Moretti, A. 2006, A\&A, 452, 179

Clarke, C. J., Bonnell, I. A., \& Hillenbrand, L. A. 2000, in Protostars and Planets IV, ed. V. Mannings, A. P. Boss, \& S. S. Russell (Tucson: Univ. Arizona Press), 151

Elson, R. A. W., Fall, S. M., \& Freeman, K. C. 1987, ApJ, 323, 54

Fall, S. M., Chandar, R., \& Whitmore, B. C. 2005, ApJ, 631, L133

Gieles, M., Larsen, S. S., Bastian, N., \& Stein, I. T. 2006a, A\&A, 450, 129

Gieles, M., Portegies Zwart, S. F., Baumgardt, H., Athanassoula, E., Lamers,

H. J. G. L. M., Sipior, M., \& Leenaarts, J. 2006b, MNRAS, 371, 793

Girardi, L., Bertelli, G., Bressan, A., Chiosi, C., Groenewegen, M. A. T., Marigo, P., Salasnich, B., \& Weiss, A. 2002, A\&A, 391, 195

Girardi, L., Bressan, A., Bertelli, G., \& Chiosi, C. 2000, A\&AS, 141, 371

Girardi, L., Bressan, A., Chiosi, C., Bertelli, G., \& Nasi, E. 1996, A\&AS, 117, 113

\section{EFERENCES}

Gnedin, O. Y., \& Ostriker, J. P. 1997, ApJ, 474, 223

Goodwin, S. P. 1997, MNRAS, 286, 669

Goodwin, S. P., \& Bastian, N. 2006, MNRAS, 373, 752

Harris, J., \& Zaritsky, D. 2004, AJ, 127, 1531

Hill, A., \& Zaritsky, D. 2006, AJ, 131, 414 (HZ06)

Hodge, P. 1987, PASP, 99, 724

Hunter, D. A., Elmegreen, B. G., Dupuy, T. J., \& Mortonson, M. 2003, AJ, 126, 1836

King, I. 1962, AJ, 67, 471

Lada, C. J., \& Lada, E. A. 2003, ARA\&A, 41, 57

Lamers, H. J. G. L. M., Gieles, M., \& Portegies Zwart, S. F. 2005, A\&A, 429, 173

Leitherer, C., et al. 1999, ApJS, 123, 3

Maíz Apellániz, J., \& Úbeda, L. 2005, ApJ, 629, 873

Mora, M. D., Larsen, S. S., \& Kissler-Patig, M. 2007, A\&A, 464, 495

Ostriker, J. P., Spitzer, L. J., \& Chevalier, R. A. 1972, ApJ, 176, L51

Rafelski, M., \& Zaritsky, D. 2005, AJ, 129, 2701 (RZ05)

Schulz, J., Fritze-v. Alvensleben, U., Möller, C. S., \& Fricke, K. J. 2002, A\&A, 392, 1

Spitzer, L. J. 1958, ApJ, 127, 17

Takahashi, K., \& Portegies Zwart, S. F. 2000, ApJ, 535, 759

Vesperini, E., \& Heggie, D. C. 1997, MNRAS, 289, 898

Whitmore, B. C., Chandar, R., \& Fall, S. M. 2007, AJ, 133, 1067

Zaritsky, D., Harris, J., \& Thompson, I. 1997, AJ, 114, 1002 